\title{
Reliability of Axial-Thoracolumbar Spine Rotation Range Measurements
}

Sung-hoon Jung, PT, Ph.D. ${ }^{1}$; Sung-min Ha, PT, Ph.D. ${ }^{2}$; Jun-hee Kim, PT, Ph.D. candidate ${ }^{1}$; Gyeong-tae Gwak, PT, BHSc ${ }^{1}$; Su-jung Kim, PT, Ph.D. ${ }^{3}$

${ }^{1}$ Physical Therapy, College of Health Science, Yonsei University, South Korea

${ }^{2}$ Department of Physical Therapy, College of Health Science, Sangii University, South Korea

${ }^{3}$ KEMA Healing Center, Daejeon, South Korea

Background Although numerous measurement methods exist for thoracolumbar spine rotation, achieving an accurate measurement of axial-thoracolumbar spine rotation is difficult because it is usually measured in the open kinematic chain.

Purpose Therefore, the purpose of our study was to investigate the reliability of a new method for measuring axial-thoracolumbar spine rotation in the closed kinematic chain.

Study design Intra-tester repeated measures

Methods 14 healthy participants performed axial-thoracolumbar rotation. An electromagnetic tracking system was used to measure the angle of axial-thoracolumbar rotation in the sternum's jugular notch and xiphoid process.

Results The test-retest reliability of the axial-thoracolumbar rotation angle per the sensor on the jugular notch was 0.83 , and the measurement from the sensor on the xiphoid process was 0.84 .

Conclusions The measurement of the axial-thoracolumbar rotation angle in the supine position showed excellent reliability.

Key words Axial-thoracolumbar rotation; Measurement; Reliability; Trunk rotation.

Journal of KEMA 2018; 2(1): 20-23 Published Online June 30, 2018 pISSN: $2586-4351$ eISSN: 2586-5706

Article History Received 18 April 2018 Revised 25 April 2018 Accepted 01 May 2018

\section{CONTACT}

sio2natu@hanmail.net Su-jung Kim, KEMA Healing Center, 74, Dunsanjung-ro, Seogu, Daejeon, South Korea

This is an Open-Access article distributed under the terms of the Creative Commons Attribution Non-Commercial $\mathrm{Li}-$ cense (http://creativecommons. org/licenses/by-nc/4.0) which permits unrestricted non-commercial use, distribution, and reproduction in any medium, provided the origin mork properly cited.

\section{INTRODUCTION}

Trunk rotation movement includes the coordinated upper and lower segment motion of the thoracolumbar spine. ${ }^{1}$ Rocket sports activities, such as tennis and golf, and daily activities require whole-thoracolumbar rotation., ${ }^{2,3}$ The thoracolumbar spine has a rotational motion approximately 40-74 degrees in the horizontal plane. ${ }^{4-6}$

To measure the thoracolumbar rotation, previous studies have used a three-dimensional motion analysis system, goniometry, and inclinometer. ${ }^{5,7-9}$ Except for the goniometry measurement, the other measurements showed excellentgood reliability and a low level of measurement error. ${ }^{9,10}$ However, a three-dimensional motion analysis system is expensive and requires considerable space. ${ }^{7}$ Therefore, cli- nicians need an easy and accurate measurement tool to measure thoracolumbar rotation.

Previous studies of measuring axial-thoracolumbar rotation have been performed in the open kinetic chain, such as the sitting or standing posture of the thoracolumbar spine without pelvis fixation. ${ }^{6,11,12}$ These methods may be difficult to measure the exact axial-thoracolumbar rotation without compensatory movement of the pelvis. To measure axialthoracolumbar rotation in the closed kinetic chain, Kim et al. (2017) measured thoraco-lumbar-pelvic rotation in the supine position with upper thorax fixation. However, there have been no studies to measure axial-thoracolumbar rotation in the closed kinetic chain. Therefore, the purpose of our study was to investigate the reliability of a new method for measuring axial-thoracolumbar rotation in the closed 
kinetic chain. We hypothesized that the reliability of the measurement method in the closed kinetic chain is high, and that there is no significant difference between testers and the measurement sections (jugular notch and xiphoid process).

\section{METHODS}

\section{Participants}

Fourteen healthy males (age $=24.43 \pm 1.95$ years; height $=$ $172.86 \pm 7.40 \mathrm{~cm}$; mass $=71.50 \pm 10.80 \mathrm{~kg}$ ) participated in this study. All study participants had measurements taken for both side (right and left) axial-thoracolumbar rotation. The participants completed a self-report to determine if there were any orthopedic or neurological problems in the upper extremities. Subjects were excluded from this study if they had (1) a history of fracture or traumatic injuries of the thoracic and lumbar spine or (2) a history of orthopedic surgery within the previous six months. All subjects received an explanation of this study and agreed to participate.

\section{Instrumentation}

A Polhemus Liberty electromagnetic tracking system (Polhe-mus, Inc., Colchester, VT, USA) was used to measure the angle of axial-thoracolumbar rotation in the horizontal plane. Two sensors were attached, one at the sternum's jugular notch and the other at the sternum's xyphoid process (Figure 1). Upper and lower trunk kinematics was obtained from a global coordinate system in the horizontal plane, with zero displacement being the start position. The angles of axial-thoracolumbar rotation were defined as the absolute angle of the thoracolumbar rotation against a fixed pelvis. Kinematic data were collected at $120 \mathrm{~Hz}$, and the mean value of the three trials was used for statistical analysis.

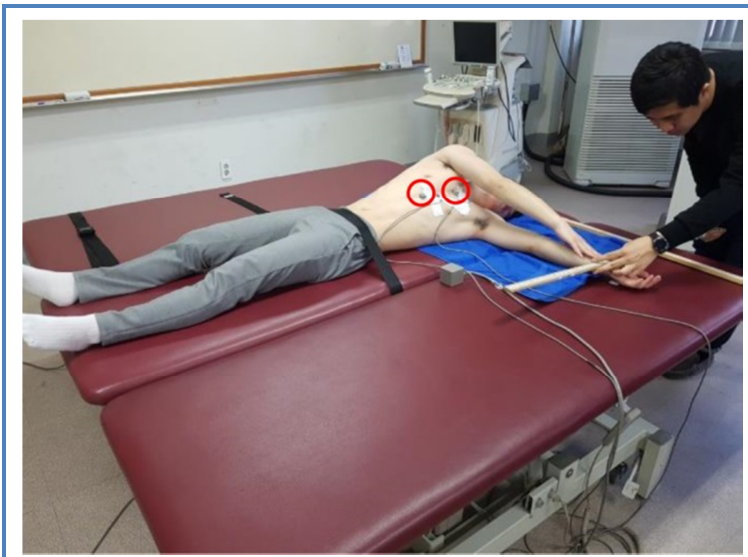

Figure 1. Sensor attachment on jugular notch and xiphoid process.

\section{Procedure}

The examiner held practice training sessions to reduce measurement error. The participant's initial position and the landmark point where a sensor was attached were the same for each session. In addition, all participants were given practice trials to familiarize them with the experimental procedures. For the axial-thoracolumbar rotation, the subjects performed an arm lateral reaching posture that abducted their arms 90 degrees in a supine position. The participant's pelvis was fixed to the table with a strap to prevent rotation. For prevent side-bending movement, the shoulder and trunk of the subject were made vertical and the subjects were instructed not to bending the trunk during arm lateral reaching. Participants were asked to rotate their thorax to reach the opposite arm against the arm in the direction of rotation (Figure 2). At this time, the arm in the direction of rotation was kept in a position where it did not fall off the floor. The point where the posture can be maintained for five seconds was measured as the axial-thoracolumbar rotation (Figure 2). The participant's performed the task twice, with a 30 -second rest period for each measurement.

\section{Statistical analysis}

A two-way analysis of variance (ANOVA) was used to test for systematic differences between the first and second tests and between the two sensors. The significance level was set at $\alpha=0.05$. The intra-test and retest reliability of the axial-thoracolumbar rotation angle were calculated using the intraclass correlation coefficient (ICC). Data were analyzed using ICC $(3,1)$ with a $95 \%$ confidence interval (CI) to determine the relative reliability of the two test sessions for axial-thoracolumbar rotation.

\section{RESULTS}

A two-way ANOVA showed no significant difference

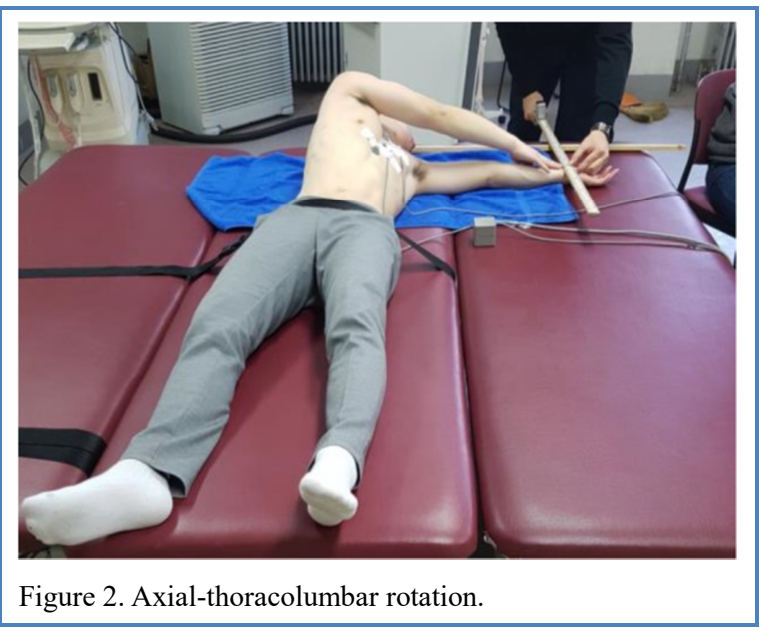


between tester and sensor location. There was no interaction between the factors. Analysis of the ICC showed the testretest reliability of the axial-thoracolumbar rotation. The test-retest ICC of axial-thoracolumbar rotation angle of the sensor on the jugular notch was 0.83 , and angle of the sensor on the xiphoid process was 0.84 (Table 1).

\section{DISCUSSION}

The purpose of this study was to evaluate the reliability of the new method for measuring axial-thoracolumbar rotation, and we found our method had high intra-rater testretest reliability (ICC: jugular notch $=0.83$, xiphoid process $=0.84)$. The measurement of the kinetic chain state in this study was a more accurate method than previous methods. Viitanen (1993) measured the axial rotation of the thoracolumbar level in a sitting posture. Johnson et al. (2012) measured the axial-thoracolumbar rotation in a sitting and halfkneeling posture. However, previous studies used a verbal comment to prevent compensation of lumbo-pelvic rotation, which may be susceptible to flexion and extension during axis-thoracolumbar rotation. ${ }^{6,13}$ Posture strongly influences thoracolumbar rotation. ${ }^{13}$ According to Edmondston et al. (2007), the angle of thoracolumbar rotation was significantly decreased in flexion compared with the neutral and extended postures. In this study, the subject's pelvis was fixed to the table with a strap to prevent compensatory movements. Furthermore, axial rotation with minimized flexion and extension could be performed compared with the open chain condition because the entire spine was a closed chain in the supine posture.

It is important to measure and improve the axialthoracolumbar rotation angle to prevent musculoskeletal pain. ${ }^{4,5,14}$ Compensated lumbo-pelvic rotation due to limited thoracolumbar rotation may cause hip joint, pelvic, and/or low back pain. ${ }^{1,14,15}$ During thoracolumbar rotation, to move ideally, the thoracolumbar spine should move in the path of instantaneous center of rotation (PICR), which is the normal amount of accessory and physiological motion available at the thoracolumbar spine. ${ }^{14}$ The lack of thoracolumbar axial rotation beyond the PICR can result in an abnormal movement pattern for the trunk during trunk rotation. ${ }^{1,14}$ Therefore, we propose this newly designed measurement method can be used for accurate axial-thoracolumbar rotation meas- urement and training in clinical practice.

The present study had several limitations. First, all participants were healthy males and relatively young subjects. These findings cannot be generalized to other populations involved in sports activities or other ages; further research is required to investigate comparisons of axis-thoracolumbar rotation in individuals with thoracolumbar dysfunctions. Second, this study investigated only intra-rater reliability. However, inter-rater test-retest reliability is also important. Further studies are needed to investigate inter-rater testretest reliability to generalize this technique. Third, in this study, we only measured the new method's reliability in the supine position to assess the axial-thoracolumbar rotation angle. Further studies are needed to investigate the validity of other task measurements using three-dimensional motion analysis system or smart phone-based measurement tool by Jung et al (2018).

\section{CONCLUSIONS}

We found excellent intra-rater test-retest reliability of the axial-thoracolumbar rotation angle measurements in the supine position. Thus, this new measurement method may provide an accurate evaluation in the clinic to measure axial-thoracolumbar rotation.

\section{Key Points}

Question What is the best way to measure axial-thoracolumbar rotation?

Findings When using the new measurement method for the axial-thoracolumbar rotation, we found excellent intra-rater test-retest reliability for the measurements of the axialthoracolumbar rotation angle in the supine position.

Meaning The new measurement method for axial-thoracolumbar rotation provides accurate evaluation in the clinic, and it may prevent compensation movement during axial-thoracolumbar rotation.

\section{Article information}

Conflict of Interest Disclosures: None.

Funding/Support: None.

Acknowledgment: None.

Table 1. Axial-thoracolumbar rotation angle reliability

\begin{tabular}{cccccc}
\hline Variable & Test 1 mean (SD) & Test 2 mean (SD) & N & ICC (3,1) (95\% CI) & $p$ \\
\hline Jugular & $41.51(13.55)$ & $39.57(14.38)$ & 28 & $0.83(0.67-0.92)$ & $<0.01$ \\
Xiphoid & $37.13(9.62)$ & $35.96(10.44)$ & 28 & $0.84(0.68-0.92)$ & $<0.01$ \\
\hline
\end{tabular}




\section{REFERENCES}

1. Kim SH, Kwon OY, Park KN, Hwang UJ. Leg lateral reach test: the reliability and correlation with thoracolumbo-pelvic rotation range. Journal of Science and Medicine in Sport. 2017;20(1):2-5.

2. Lindsay D, Horton J. Comparison of spine motion in elite golfers with and without low back pain. Journal of Sports Sciences. 2002;20(8):599-605.

3. Aguinaldo AL, Buttermore J, Chambers H. Effects of upper trunk rotation on shoulder joint torque among baseball pitchers of various levels. Journal of Applied Biomechanics. 2007;23(1):42-51.

4. Neumann DA. Kinesiology of the musculoskeletal systeme-book: foundations for rehabilitation. Elsevier Health Sciences; 2013.

5. Viitanen J. Thoracolumbar rotation in ankylosing spondylitis. A new noninvasive measurement method. Spine. 1993;18(7): 880-883.

6. Johnson KD, Kim KM, Yu BK, Saliba SA, Grindstaff TL. Reliability of thoracic spine rotation range-of-motion measurements in healthy adults. Journal of Athletic Training. 2012;47(1):52-60.

7. Intolo $\mathrm{P}$, Carman $\mathrm{AB}$, Milosavljevic $\mathrm{S}$, Abbott JH, Baxter GD. The spineangel ${ }^{\circledR}$ : Examining the validity and reliability of a novel clinical device for monitoring trunk motion. Manual Therapy. 2010;15(2):160-166.

8. Mannion A, Troke M. A comparison of two motion analysis devices used in the measurement of lumbar spinal mobility. Clinical Biomechanics. 1999;14(9):612619.
9. Kachingwe AF, Phillips BJ. Inter-and intrarater reliability of a back range of motion instrument. Archives of Physical Medicine and Rehabilitation. 2005;86(12):2347-2353.

10. Montgomery T, Boocock M, Hing W. The effects of spinal posture and pelvic fixation on trunk rotation range of motion. Clinical Biomechanics. 2011;26(7): 707-712.

11. Dillard J, Trafimow J, Andersson G, Cronin K. Motion of the lumbar spine. Reliability of two measurement techniques. Spine. 1991;16(3):321-324.

12. Mellin G. Method and instrument for noninvasive measurements of thoracolumbar rotation. Spine. 1987; 12(1):28-31.

13. Edmondston SJ, Aggerholm M, Elfving S, et al. Influence of posture on the range of axial rotation and coupled lateral flexion of the thoracic spine. Journal of Manipulative \& Physiological Therapeutics. 2007;30(3): 193-199.

14. Sahrmann S. Movement system impairment syndromes of the extremities, cervical and thoracic spines-e-book. Elsevier Health Sciences; 2010.

15. Sahrmann S. Diagnosis and treatment of movement impairment syndromes. Elsevier Health Sciences; 2002.

16. Jung SH, Kwon OY, Jeon IC, Hwang UJ, Weon JH. Reliability and criterion validity of measurements using a smart phone-based measurement tool for the transverse rotation angle of the pelvis during single-leg lifting. Physiotherapy Theory and Practice. 2018;34(1):5865. 